\title{
Coopetition Spectrum Trading in Cognitive Radio Networks
}

\author{
Liu Cui \\ School of Information Sciences \\ University of Pittsburgh \\ Pittsburgh, PA 15232 \\ Email: lic49@pitt.edu
}

\author{
Taieb Znati \\ Department of Computer Sciences \\ University of Pittsburgh \\ Pittsburgh, PA 15232 \\ Email: znati@cs.pitt.edu
}

\begin{abstract}
Spectrum trading is a promising method to improve spectrum usage efficiency. Several issues must be addressed, however, to enable spectrum trading that goes beyond conservative trading idle bands and achieve cooperation between primary and secondary users. In this paper, we argue that spectrum holes should be explicitly endogenous and negotiated by spectrum trading participants. To this end, we proposed an a Vickery auction based, coopetive framework to foster cooperation, while allowing competition for spectrum sharing. Incentive schemes and penalty for revocable spectrum are proposed to increase the spectrum access opportunities for SUs while protecting PUs spectrum value. A simultation study shows that the proposed framework outperforms conservative trading approaches, in a variety of scenarios with different levels of cooperation and bidding strategies.
\end{abstract}

\section{INTRODUCTION}

Cognitive radios, with their ability to sense and dynamically adjust their operating parameters to operational environment, have emerged as a key enabling technology of Dynamic Spectrum Access (DSA) to harness unused spectrum and achieve efficient management of spectrum resources [1]. There are two basic methods to implement DSA: opportunistic access and cooperative sharing. In opportunistic access, secondary users (SUs) sense the spectrum environment and make autonomous decisions on spectrum access, cognizant of the fact that they must vacate the spectrum upon return of the primary user (PU). On the other hand, cooperative sharing requires coordination between PUs and SUs to achieve efficient spectrum access. The focus of this paper is on cooperative sharing, which shows greater potential in terms of supporting users Quality of Services (QoS) guarantees. More specifically, we consider cooperative sharing through spectrum trading.

While conceptually simple, the realization of cooperative DSA gives rise to several technical and regulatory challenges. Technical challenges stem from the inherent difficulty of accurately sensing radio environments and efficiently coordinating transmission activities. The regulatory issues deal with the need to certify and enforce compliance with the defined access rights.

A class of cooperative spectrum sharing schemes proposes market-based approaches for efficient spectrum sharing [2]. Several of these schemes focus on analyzing spectrum trading using game theoretic- and auction-based frameworks. In these contexts, the spectrum sharing mechanisms result from the induced dynamics of the Cognitive Radio Networks(CRN). Game theoretic approaches aim at finding the best strategies that optimize the utilities for PUs and SUs, in different scenarios [2].Auction-based approaches view CRN resources as divisible goods in order to maximize the total utility of the competing parties [3] [4].

The intense research in DSA has led to several schemes for efficient spectrum management and allocation in CRNs. Most of these schemes, however, use questionable assumptions that limit their applicability in a real setting. For example, in both opportunistic and cooperative schemes, spectrum holes are considered to be exogenous. This implies SUs cannot control the spectrum hole and PUs' behavior is invariant to the presence of SUs. In this paper, we argue that spectrum holes should be explicitly endogenous. Consequently, spectrum holes are created as the result of bilateral bargaining on the part PUs and SUs, or they may occur through a broker or a transaction in a spectrum market. To this end, we propose a coopetitve DSA framework, to enable efficient spectrum trading by fostering cooperation, while allowing competition. The main contribution of this paper are: (1) incentives for creating endogenous spectrum holes; (2) a revocable leasing scheme to mitigate the risks of PUs and SUs in trading spectrum; and (3) a spectrum auction to enable competitive spectrum trading. A simulation study is performed to assess coopetive spectrum sharing framework in achieving efficient spectrum management. The results show that both PUs and SUs benefit from coopetitive spectrum trading.

The rest of the paper is organized as follows: Section II introduces the proposed spectrum trading framework, including two incentive schemes and the utility for each market participant. Section III provides the numerical results and analysis. Section IV concludes the paper the present future researches.

\section{COOPETITION FRAMEWORK}

The cooperative framework establishes a dynamic auction driven-market, to assign spectrum to those who value it the most. In the following, we describe the goals and objective of the framework. We also describe the auction structure, 
participants, and mechanisms, including the incentives which are designed to maximize the expected utility of PUs and SUs.

\section{A. Objectives and goals}

To manage spectrum efficiently, PUs must determine the number of channels to support their internal services and the number of channels that can be leased to SUs. It is, however, difficult to accurately predict service demand a priory, especially for an extended period of time. PUs may adopt a conservative strategy, whereby they over-reserve channels for their internal use and only make a minimum number of channels available to SUs. An aggressive PU, on the other hand, may opt to increase the number of leaseable channel at the risk of service degradation. Neither of these strategies is effective. What is needed is an incentive-based, risk-mitigating framework, whereby spectrum holes are created by encouraging PUs to increase the leaseable channels, while limiting the risk of service degradation. Such a framework must provide the following:

- Mechanisms to incentivize PUs to increase leasable spectrum channels, while minimizing the risk of service degradation.

- Mechanisms to deter PUs from revoking leased channels before the expiration of the leasing period.

- Mechanisms to encourage SUs to compete for spectrum acquisition, while cooperating to mitigate their risk and the risk of the PUs, and

- Mechanisms to prevent SUs from intentionally hoarding spectrum to gain unfair advantage in the trading marked.

To meet these requirements, the coopetive framework uses Vickrey auction to enable competition through bidding. We further augment the auction with incentive and revocableleasing agreements to encourage collaboration among the auction participants, mitigate their risks and maximize their expected utilities.

\section{B. Auction and participants}

Auctions have emerged as a useful tool for spectrum management. In this framework, we adopt a multi-unit sealedbid secondary price auction, also referred to Vickrey auction. Participants in Vickrey auctions simultaneously submit bids. The auctioneer disclose the identity of the highest bidder, who is declared the winner. The price paid, however, is equal to the second highest bid placed by the participants. Truth-telling is enforced in Vickrey auction; bidders, therefore have no incentive to manipulate the bids to increase their utilities [5].

The coopetive framework auction is designed as a set of trading epochs, coordinated by a broker. During each epoch PUs and SUs engage in trading spectrum channels. PUs are incumbent spectrum licensees, with different preferences, who can be risk averse or prone. Furthermore, we assume that the value PUs derive from their licensed spectrum depends on their ability to generate revenue. PUs can generate revenue either by using their entire spectrum, or part thereof, for their mostvalued service. They can also entirely or partially lease their spectrum to other entities for their own use. It is assumed that

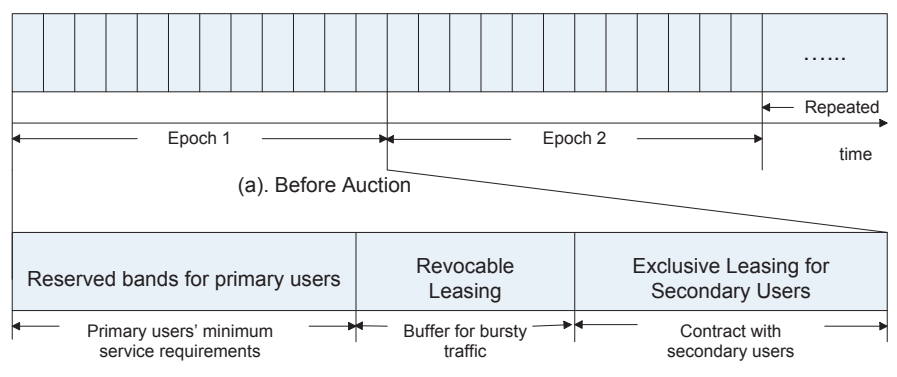

(b). After Auction

Fig. 1. Primary User Spectrum Structure.

the PUs spectrum utility simultaneously captures the revenue generated from trading leased channels and the risk-aversion associated with leasing these channels. A rational PU seeks to find an optimal partition of licensed channels to maximize the expected utility.

SUs seek to acquire PUs' licensed spectrum for specified time periods either to support their most valued internal applications or use these channels to satisfy service demands of their user communities. It also is assumed that the SUs spectrum utility function takes into consideration both the revenue and potential risk of leasing revocable PUs'channels.

The trading activities between PUs and SUs are coordinated by a spectrum broker. The responsibilities of the broker include matching supply and demand for PUs and SUs, facilitating spectrum trading transactions, allocating spectrum according to demand and usage, and coordinating interference among different systems. The role of spectrum broker can be conducted by an organization or carried out through a database [6].

At the completion of the coopetive auction, PUs' licensed channels can be classified into three categories: PU reserved $(p R)$, exclusively-leased $(e L)$, and revocably-leased $(r L)$. The $p R$ channels are exclusively reserved for PUs' access to support QoS requirements of the provided wireless services. The $e L$ channels are exclusively dedicated to SUs to support their service demands over the entire trading epoch. The $r L$ channels provide PUs with the opportunity to increase QoS and meet bursty traffic demand by revoking the spectrum when needed during trading epochs. An instance of a PU's spectrum structure is depicted in Fig.1.

\section{Incentive for Creating Spectrum Holes}

PUs and SUs trade spectrum under uncertainty.To reduce risk aversion, the coopetive framework uses revocable leasing and penalties. The revocable leasing mechanism provides an incentive for PUs to make more spectrum available for trade, as it mitigates their risk of unforeseen increase of the spectrum demands of their most-valued services. PUs revoke the spectrum by sending signals to SUs. When receiving this message, SUs are obligated to vacate the spectrum.

Penalties deter both parties from gaming the system to gain unfair advantage in trading spectrum. In order to protect SUs 
investment and prevent abuse of spectrum revocation, PUs seeking to revoke spectrum must pay a penalty. The terms of the penalty are negotiated bilaterally by PUs and SUs and enforced based on the binding final agreement. The penalty can be in the form of monetary funds or in-kind benefits.

\section{Ranking for Cooperative Trading}

In order to foster cooperative spectrum trading, the coopetive framework uses a ranking system to encourage SUs to voluntarily vacate revocable spectrum. By voluntarily relinquishing revocable spectrum, SUs increase their ranks in PUs' preferred traders' list. The rank, combined with the bidding price, is used to determine the winner among all competing SUs for spectrum access.

In the coopetive framework, the broker uses a scoring function and maintains a reward database to keep track of the points PUs award to SUs who voluntarily vacate their licensed channels. The reward score achieved by an SU in epoch $\tau$ determines their ranking in epoch $\tau+1$. SUs with higher ranking are likely to succeed in acquiring spectrum.

SUs' decision to voluntarily relinquish spectrum depends on the balance between their long-term spectrum profits and temporary service loss. $S U_{i}$ 's cost, in terms of QoS degradation or service termination, is expressed in Eq.(1), where $V_{i j}^{k}$ is $S U_{i}$ 's spectrum value for $P U_{j}$ 's channel $k$, and $Q_{i j}^{e}$ is the number of channels that $S U_{i}$ evacuate for $P U_{j}$.

$$
\text { Cost }_{i}=\sum_{j=1}^{m} \sum_{k=1}^{Q_{i j}^{e}} V_{i j}^{k},
$$

Several strategies can be used by SUs to determine their level of cooperation with PUs. In this paper, we focus on three strategies, namely myopic, short-term, and long-term. SUs with myopic strategy only consider next term profit. SUs with short-term strategy consider the profit for a limited number of terms. SUs with long-term strategy consider profits for an extended period of time. We use the net present value (NPV) as a valuation methodology to compare the cooperative and noncooperative strategies. $S U_{i}$ 's NPV is expressed in Eq.(2), where $Y$ indicates the total number of trading epochs that $S U_{i}$ considers. int is the interest rate, $E\left(U_{S i}^{y}\right)$ is $S U_{i}$ 's expected utility at $y^{\text {th }}$ trading epoch.

$$
N P V_{i}=\sum_{y=1}^{Y} \frac{E\left(U_{S i}^{y}\right)}{(1+i n t)^{y}},
$$

The decision to evacuate revocable channels depends on the NPV value of each strategy.

\section{E. Auction Utilities}

1) Primary Users: In each auction epoch, PUs provide the broker with their expected channel trading price. The same PU may have different prices for variance bands. A bid lower than PUs' expected price translates into a negative for the PU. Such an impact manifests itself in the form of QoS degradation, customer loss, etc. The expected price vector for $P U_{j}$ is defined by PUs' as $E P_{j}^{k}=\left(E P_{j}^{1}, E P_{j}^{2}, \cdots, E P_{j}^{K}\right)$, where $k$ indicates the number of channel. The utility function of $P U_{j}$ can then be expressed by Eq.(3), where $C_{j}^{k}$ is the amount of money that SUs pay for $P U_{j}$ 's channel $k, K^{\prime}$ is the number of channels that are traded, and $\alpha_{j}\left(0<\alpha_{j}<1\right)$ is the broker's share of leasing revenue.

$$
U_{j}^{p}=\left(1-\alpha_{j}\right) \sum_{k=1}^{K^{\prime}} C_{j}^{k}-\sum_{k=1}^{K^{\prime}} E P_{j}^{k}-E\left[F_{j}\right],
$$

The first part of PU's utility represents the revenue generated from spectrum leasing. The second part describes PUs' potential service loss incurred by spectrum leasing. The third part is the expected value of the penalty for revoking spectrum from SUs.

PUs' objective is to maximize its utility, and can be formulazied as:

$$
\begin{array}{lc}
\text { maximize } & U_{j}^{p} \\
\text { subject to } & C_{j}^{k} \geq E P_{j}^{k}
\end{array}
$$

The constraint guarantees that all spectrum cost is higher than PUs' expected price. The simulation, provided in section III, shows the PUs' profit in different scenarios, using the utility function described above.

2) Secondary Users: The spectrum value for SUs depends on several characteristics, including bandwidth, frequency, modulation, and geographical location. We assume that channels from the same PU are identical, and, therefore, the spectrum value a SU associates with channels from the same PU only depends on the relationship between utility and bandwidth. Depending on SUs' services, four types of utilities can be defined, namely elastic, real-time, rateadaptive and stepwise [7]. We assume that SUs' spectrum value is a stepwise function, and use different parameters to reflect different SU's utilities. We further assume that $S U_{i}$ 's spectrum value for $P U_{j}$ 's channel $k$, is defined as $V_{i j}^{k}=g\left(w_{i}\right)^{k}$, where $g()$ is a nonnegative and nondecreasing function of the channel bandwidth $w_{i}$. Consequently, $S U_{i}$ 's value matrix for the selected channels from each $\mathrm{PU}$ is $W_{i}=\left(V_{i 1}^{1}, \cdots, V_{i 1}^{K} ; V_{i 2}^{1}, \cdots, V_{i 2}^{K} ; \cdots ; V_{i n}^{1}, \cdots, V_{i n}^{K}\right)$. SUs set the value of the remaining channels to 0 . We assume that SUs are rational entities who aim at maximizing their own utility and, therefore, bid according to spectrum true value.

The SU's utility function is expressed in Eq.(6), where $C_{i j}^{k}$ denotes $S U_{i}$ spectrum cost for $P U_{j}$ 's channel $k$, which is determined by the losing bid price, and $k_{j}$ is the number of channels that $S U_{i}$ lease from $P U_{j}$.

$$
U_{i}^{S}=\sum_{j=1}^{n} \sum_{k=1}^{k_{j}}\left(V_{i j}^{k}-C_{i j}^{k}\right)-E\left(C_{R}\right)+E(F),
$$

The first part of SU's utility represents the profit gained from services provided by the acquired leased channels. The second part represents the service loss incurred by spectrum revocation, while the third represents the compensation gained 
from relinquishing a channel to its original PU. The SU's utility optimization can be expressed as:

$$
\begin{array}{lc}
\operatorname{maximize} & U_{i}^{S} \\
\text { subject to } & V_{i j}^{k} \geq E P_{j}^{k}
\end{array}
$$

3) The Broker: The broker's utility function, expressed in Eq.(9), comprises three components. The first, $C_{i j}^{k}$, represents the partial revenue from spectrum leasing,derived from trading $P U_{i}$ trading channel $k$ to $S U_{j}(1<=i<=\widehat{n})$, where $\widehat{n}$ is the number of active PUs and $\widehat{K}$ is the total number of channels traded by $P U_{j}$. The second part is the trading cost, $C_{t}$, collected from $S U_{j}(1<=j<\widehat{m})$, where $\widehat{m}$ is the number of active SUs. The third part, $\left(C_{o}\right)$, is the broker's operating cost.

$$
U^{B}=\sum_{j=1}^{\widehat{n}} \sum_{i=1}^{\widehat{m}} \sum_{k=1}^{\widehat{K}} \alpha_{j} \cdot C_{i j}^{k}+\sum_{i=1}^{\widehat{m}} C_{t}-C_{o}
$$

The above utility function reflects the broker's interest to encourage and foster collaborative and successful trading.

\section{Spectrum Allocation And Analysis}

. In the following we describe the scheme the broker uses to allocate channels to bidders. We then discuss a simulationstudy to assess the performance of coopetive framework in fostering collaboration, while allowing competition. We limit the analysis to the case of multiple SUs and only one PUs.

\section{A. Spectrum Allocation}

During each epoch, the broker ranks SUs based on (1) SU's spectrum bid, and (2) PUs' preference of SUs. For a given PU, we use $R_{i k}^{b}$ to denote $S U_{i}$ 's bid for the $k^{t h}$ requested band, and $R_{i}^{c}$ to denote $S U_{i}$ 's ranking with respect to PUs preference. The $R_{i}^{c}$ value is shown in Eq. (10), where, $R_{i}(\tau)$ is the reward point for SUs at trading epoch $\tau$, and $R_{\min }(\tau)$ and $R_{\max }(\tau)$ are the minimum and maximum reward point in period $\tau$, respectively.

$$
R_{i}^{c}=\frac{R_{i}(\tau)-R_{\min }(\tau)}{R_{\max }(\tau)-R_{\min }(\tau)}
$$

The final rank is calculated as shown Eq.(11).

$$
R_{i k}^{f}(\tau+1)=\beta \cdot R_{i k}^{p}(\tau+1)+(1-\beta) \cdot R_{i}^{c}(\tau)
$$

The parameter $0 \leq \beta \leq 1$ is a weight factor to indicate the importance of each ranking method.

The broker assigns the bands based as follows: The overall highest rank, obtained by combining SUs' bids with PUs' preference, is allocated one band. The winner, however, still pays the price of the highest loosing bid. The second highest rank is allocated the next band. This process continues until either the channel supply or demand have been met.

\section{B. Spectrum Analysis}

In order to gain better understanding of the coopertive framework potential to achieve an efficient spectrum management, Matlab is used to simulate spectrum trading in different scenarios. Fig. 2 shows the spectrum allocation for one trading epoch of a PU who has 10 identical wireless channels, under 7 different scenarios. Fig.3 depicts the comparison of PUs' expected spectrum income using the proposed framework and the conservative approach where PUs only lease idle bands. Factors that impact spectrum allocation and PUs' profits include: (1) PU's spectrum utilization; (2) SU's proposed spectrum price; (3) the level of penalty; and (4) cooperation between PUs and SUs. In Fig.2, blue represents the number of channels reserved for primary users, green indicates the number of bands that are revocably leased to SUs, and red describes bands that are exclusively leased to SUs. In Fig.3, blue bars illustrate profits gained by PUs in the coopetive framework, while red bars show the profits gained by PUs by selling only idle bands, for different scenarios. The first scenario, bench, is the benchmark case, in which the PU needs spectrum $40 \%$ of the time, and SUs do not voluntarily vacate the frequency bands when PU needs them. The penalty of revoking spectrum is assumed to be equal to the spectrum cost. Additional scenarios are derived from the benchmark case to reflect different levels of collaboration, namely full and partial collaboration, labeled as full, part, respectively. In full, SUs voluntarily vacate the channel $100 \%$ of the time, while in part the channel is only vacated $80 \%$ of the time. We also study the impact of the penalty on collaboration, by reducing its value to $80 \%$ of the spectrum cost, in the less scenario. In the difpri $_{n}$ and difpri $_{c}$ scenarios, SUs' proposed spectrum price is reduced to $80 \%$ of the benchmark value. In difpri $i_{n}$, SUs do not cooperate with the PU, while in difpri $_{c}$ SUs fully cooperate. In the final scenario, PU's service demand increases from $40 \%$ to $60 \%$.

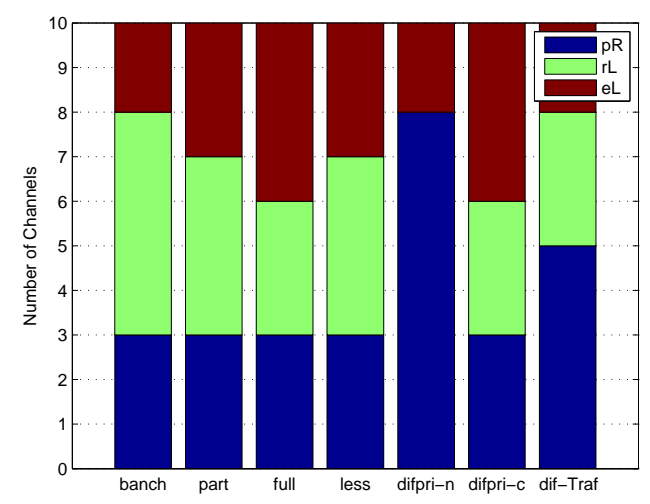

Fig. 2. Spectrum Allocation for Trading.

Several observations can be drawn from the results depicted in Fig.2. First, PUs are more aggressive in leasing the spectrum when the penalty decreases. Second, when SUs' bid decreases, revocable leasing is no longer attractive to PUs, as the spec- 
trum leasing gain no longer balances the potential penalty risk. Third, the leaseable spectrum decreases when PUs' service demand increases. Furthermore, the results show that the incentive mechanisms of the coopetive framework lead to a more efficient spectrum management than the conservative, opportunistic spectrum sharing. In all scenarios, the proposed framework achieves higher profits than the conservative framework, as depicted in Fig.3. Only in the case where the SU's bid decreases that both approaches achieve the same profits. The results clearly demonstrate that the cooperation between PUs and SUs increases PUs' profits, while meeting PU's service requirements through spectrum revocation. This is mostly due to reducing PU's aversion to risk.

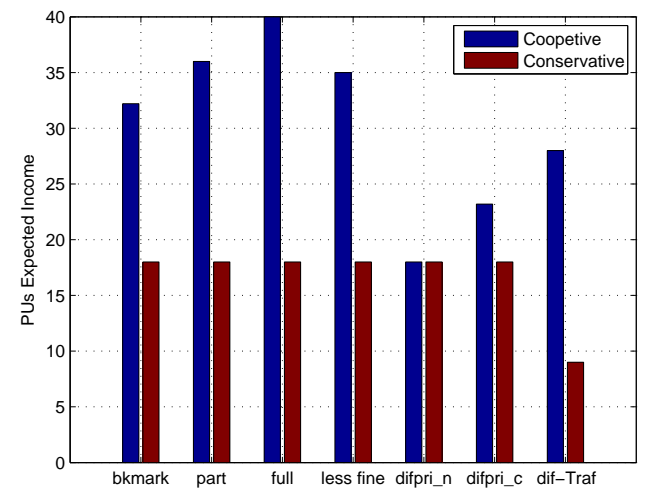

Fig. 3. Comparison of PUs' Expected spectrum income.

\section{Cooperative Scenario Analysis}

In this part of the analysis, we focus on the case where SUs voluntarily vacate the leased channels, when requested by the PUs. We assume that SUs lease three channels and PU can revoke up to three channels. Three major factors impact SUs' decision: namely the SUs' spectrum value, the desire of SUs to increase their cooperative score, and the SUs' current rank. We conduct simulation experiments, where each impact factor is set to high, $H$, or low, $L$. We further consider three strategies, myopic, short-term and long-term.

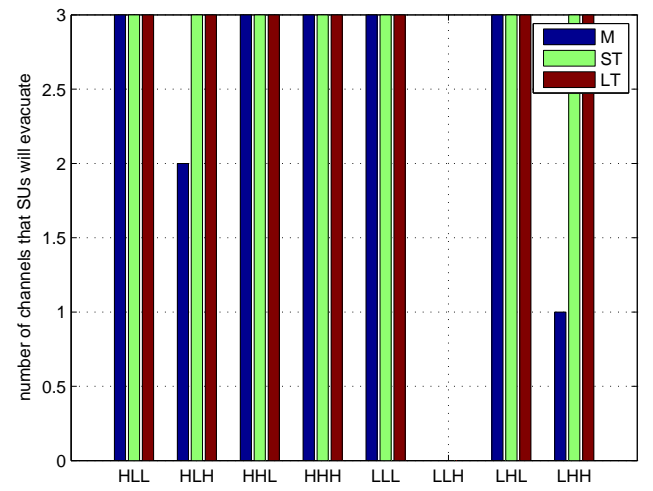

Fig. 4. Situations for SUs to Evacuate the channels.
Several observations can be drawn from the results, depicted in Fig.4, where blue, green, and red bars represent myopic, the short-term, and long-term strategies, respectively. First, SUs with long-term strategies are more likely to cooperate with PUs. Second, if SU's ranking is already high, they are less likely to vacate the channel voluntarily. Third, when the score increment is high, SUs are more likely to free up the spectrum. Fourth, when spectrum values are high, SUs' collaboration with PUs is no longer profitable; SUs win exclusive access to channels through increased bids due to high score.

\section{CONCLUSION}

Spectrum market is essential to achieve efficient spectrum management and increase PUs and SUs utilities. In this paper, we propose a coopetive framework which allows competition and promotes cooperation between PUs and SUs. Two incentives are implemented to achieve this goal. The first, is a revocable lease mechanism, which encourages PUs to increase endogenous spectrum holes. A penalty is used to prevent PUs from revoking spectrum in order to avoid competition and intentionally degrade SUs' services. The second incentive is a scoring function that encourages SUs to cooperate with PUs. SUs' decision relies on the balance between long-term profits and current service loss. It also depends on SUs' perception of expected gain.

A simulation-based analysis shows that the coopetive framework achieves higher profits than the classic conservative spectrum sharing framework. The framework also enhances SUs' ability to get access to spectrum, in multiple scenarios with varying levels of cooperation and competition. Future work will focus on different PUs' traffic model and QoS requirements, different SUs' utility functions that go beyond stepwise utilities, and different mechanisms for cooperation among risk-averse and risk-prone users.

\section{REFERENCES}

[1] I. F. Akyildiz, W.-Y. Lee, M. C. Vuran, and S. Mohanty, "Next generation/dynamic spectrum access/cognitive radio wireless networks: A survey," COMPUTER NETWORKS JOURNAL (ELSEVIER, vol. 50, pp. 2127-2159, 2006.

[2] K. R. Liu and B. Wang, Cognitive radio networking and security: A game-theoretic view. Cambridge University Press, 2010.

[3] S. Gandhi et al., "A general framework for wireless spectrum auctions," in New Frontiers in Dynamic Spectrum Access Networks, 2007. DySPAN 2007. 2007 2nd IEEE International Symposium on, April 2007, pp. 2233.

[4] J. Huang, R. A. Berry, and M. L. Honig, "Auction-based spectrum sharing," Mob. Netw. Appl., vol. 11, no. 3, pp. 405-418, Jun. 2006. [Online]. Available: http://dx.doi.org/10.1007/s11036-006-5192-y

[5] V. Rodriguez, K. Moessner, and R. Tafazolli, "Auction driven dynamic spectrum allocation: Optimal bidding, pricing and service priorities for multi-rate, multi-class cdma," in PIMRC 2005. The 16th International Symposium on Personal, Indoor and Mobile Radio Communications. IEEE, vol. 3, Sept. 2005, pp. $1850-1854$.

[6] S. Im and H. Lee, "Dynamic spectrum allocation based on binary integer programming under interference graph," in Personal Indoor and Mobile Radio Communications (PIMRC), 2012 IEEE 23rd International Symposium on. IEEE, 2012, pp. 226-231.

[7] Z. Cao and E. W. Zegura, "Utility max-min: An application-oriented bandwidth allocation scheme," in INFOCOM 1999. The 18th Conference on Computer Communications. IEEE, vol. 2, march 1999, pp. 793 -801. 